\title{
Energetics and Stability of Nanostructured Amorphous Carbon
}

\author{
M.G. Fyta, I.N. Remediakis, and P.C. Kelires \\ Physics Department University of Crete, P.O. Box 2208, 710 03, Heraclion, Crete, Greece
}

\begin{abstract}
Monte Carlo simulations, supplemented by ab initio calculations, shed light into the energetics and thermodynamic stability of nanostructured amorphous carbon. The interaction of the embedded nanocrystals with the host amorphous matrix is shown to determine in a large degree the stability and the relative energy differences among carbon phases. Diamonds are stable structures in matrices with $\mathrm{sp}^{3}$ fraction over $60 \%$. Schwarzites are stable in low-coordinated networks. Other $\mathrm{sp}^{2}$-bonded structures are metastable.
\end{abstract}

\section{INTRODUCTION}

Interest on nanostructured amorphous carbon (na-C) is steadily growing for its properties, both mechanical and electronic, that will supplement those of the traditional, single-phase a-C. Na-C can be described as a composite material in which carbon nanocrystallites, of various sizes and phases, are embedded in the a-C matrix. This novel hybrid form of carbon offers the unique possibility to intermingle the properties of carbon nanostructures $\frac{1}{\underline{\underline{m}}}$ with those of pure a-C ${ }^{2.3}$ For example, since some of these nanostructures are proposed to be insulating, while others to be metallic, the possibility is opened for tailoring the electronic properties of a-C by controlling the type and size of the embedded structures.

$\mathrm{Na}-\mathrm{C}$ films can be composed by a variety of carbon nanostructures, ranging from diamond crystallites, to concentricshell graphitic onions 1 bound by van der Waals (VDW) forces, to entirely three-dimensional $\mathrm{sp}^{2}$ covalent conformations with no VDW bonding. The latter include porous, open graphene structures with negative curvature

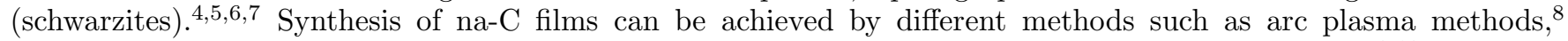

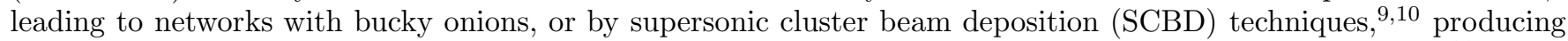
schwarzites, or by electron irradiation of a-C, producing onions and eventually nucleation of diamond cores 11

Significant insight was gained through these experiments but several issues about the structure, energetics and stability of na-C are not yet clear. Theoretical work up to now has been mainly focused on the free-standing periodic

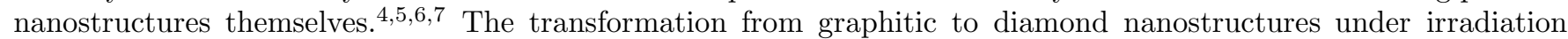
conditions is also well studied $\stackrel{11}{\underline{1}}$ However, knowledge about the interaction of the embedded nanostructures with the host amorphous matrix is very limited. This interaction is a crucial factor since it determines the stability of a given nanostructure. Variations of the density and coordination of the amorphous matrix are expected to control the stability and the relative energy differences among the carbon phases.

Here, we propose a novel theoretical approach to the problem of energetics and stability of na-C, which aims at unraveling the fundamental principles governing the interaction of nanostructures with the a-C matrix. It is based on Monte Carlo (MC) simulations within the empirical potential method. This approach is not limited to a-C, but can be applied to any nanostructured material. It is a general method, no matter which energy functional is used. For each computer-generated nanostructure embedded in the amorphous matrix, with varying coordination $z_{a m}$, its formation energy $E_{\text {form }}$, interfacial geometry, density, and stress state were calculated. Several interesting conclusions follow this analysis. For example, the question whether diamonds and schwarzites are stable in the amorphous matrix is a crucial one and of wide interest, and we give answer to that for the first time.

In the following, we first describe our theoretical methods. We then explain how we generate periodic cells with various nanostructures embedded in a-C matrices, we analyze the energetics by means of their formation energy, we study their stability as a function of size, temperature and coordination of the matrix, and we consider the possibility of transformations from one nanophase to another. Finally, we give our conclusions and prospects for future work.

\section{METHODOLOGY}

The simulations have been carried out using the empirical potential of Tersoff, 12 which provides a fairly good description of the structure and energetics of a wide range of a-C phases ${ }^{13.14}$ It was recently used in a similar context by Donadio et al $\underline{10}^{10}$ in molecular dynamics simulations of the growth of SCBD films. It was nicely shown that pore structures resembling schwarzites can be formed. The important question, to which we answer below, is whether these are stable or metastable, and at what conditions.

A limitation in the model is the absence of dispersion (VDW) forces, similar to more refined tight-binding or first-principles methods, which makes the study of structures involving VDW bonding not possible. There also is an absence of repulsion between non-bonded $\pi$ orbitals ${ }^{15,16,17}$ leading to the overestimation of density for a given $\mathrm{sp}^{3}$ 
content. 14.18 This mostly occurs at intermediate $\mathrm{sp}^{3}$ contents, while low-sp ${ }^{3}$ (evaporated a-C) and high-sp ${ }^{3}$ (ta-C) networks, are properly described in this respect. For ta-C $\left(\mathrm{sp}^{3}\right.$ fraction about $\left.80 \%\right)$ the overestimation is $\sim 3-4 \%$. We do not expect this shortcoming to seriously affect the energetics of the amorphous matrix, because this is determined primarily by the $\sigma$ bond character of the network, which is very well treated by the potential. The absence of $\pi$ repulsion has only a secondary effect on the energetics, through the generation of denser networks at intermediate contents, not serious enough to change the trends reported here.

Since the system under study is a composite material, and crystalline nanograins are part of it, it is necessary to show that the empirical potential is adequate in describing these phases. So, in order to establish the reliability of the potential for the present problem, we calibrated and checked it with respect to the energetics of various crystalline carbon structures by comparing to ab initio calculations. For the latter, we used the Vienna Ab-initio Simulation Package (VASP) code 19 We selected a number of structures that are each one of them representative of a specific group of conformations, and which are subsequently embbeded in amorphous matrices: (a) Diamond (D); (b) the BC8 structure for carbon (internal parameter $x=0.0937$ ), fourfold-coordinated with high density, a high-pressure phase; (c) polybenzene $\left(6.8^{2} D\right)$, a threefold-coordinated structure with negative curvature, and with a $D$ periodic minimal surface, that can be described as a three-dimensional linkage of benzenelike rings 6 ; (d) another sp $^{2}$-bonded, negative-curvature structure, $6.8^{2} P$, with a $P$ minimal surface, described as a condensation of truncated-icosahedral $\mathrm{C}_{60}$ molecules ${ }^{6}$; (e) PCCM (porous conducting carbon modification), an $\mathrm{sp}^{2}$-bonded structure with eightfold rings, described as a dense packing of compressed nanotubes with shared walls ${ }^{7}$; and (f) $\mathrm{C}_{168}$, the low-density, negativecurvature schwarzite structure ("buckygym") proposed by Vanderbilt and Tersoff $\stackrel{4}{\underline{4}}$ Structures (c)-(f) do not involve VDW bonding. Onionlike shell structures bound by VDW forces can not be treated with the present potential. However, Polybenzene, $6.8^{2} \mathrm{P}$, and PCCM can be considered as candidate structures resulting from fragmentation and distortion of embedded fullerenes and nanotubes in amorphous matrices. There is extensive experimental research on this method of producing composite carbon films $\stackrel{20,21,22,23}{=}$

The energies of these structures relative to diamond as a function of the density are given in Fig. 1. Our $a b$ initio energies for $6.8^{2} D, 6.8^{2} \mathrm{P}, \mathrm{BC} 8$, and $\mathrm{PCCM}$ are consistent with previous results $\underline{6.7}$ Overall, there is very good agreement between the empirical and the ab initio calculations both with respect to the energies and the density of the structures, providing confidence in using this potential for the investigation of na-C. The agreement is particularly good for the $\mathrm{C}_{168}$ schwarzite. Only in the case of PCCM a noticeable relative energy difference exists, but it does not affect the trends in our investigations. The lowest energy structure is polybenzene, followed closely by PCCM and $\mathrm{C}_{168}$. The highest energy structure is $\mathrm{BC} 8$, as expected, since it is a high-pressure phase.

\section{RESULTS AND DISCUSSION}

\section{A. Generation of structures}

The embedded nanostructures are formed by MC melting and subsequent quenching at constant volume the corresponding crystal structures, while keeping a certain number of atoms in the central portion of the cells frozen in their ideal crystal positions. Periodic boundary conditions are applied to the cells. The total number of atoms ranges from $\sim 2500$ to 4096, depending on the structure, while the number of atoms in the nanocrystals ranges from $\sim 300$ to 500 .

After quenching, which produces amorphization of the surrounding matrix, the cells are thoroughly relaxed with respect to atom positions and density. Relaxations are particularly important at the interface region, where the crystallites mainly adjust to the host environment. Cells with varying coordination (density) of the amorphous matrix can be formed by changing the initial starting density (volume) of the crystal structures. The size (radius ) of the nanocrystals is controlled by the choice of the number of the shells kept frozen during quenching. Their shape depends on the particular crystal symmetry.

Four of the generated composite cells with embedded nanostructures are illustrated in Fig. 2. In these snapshots, diamond is embedded in a highly tetrahedral matrix $\left(z_{a m} \simeq 3.8\right)$, polybenzene and PCCM are embedded in a typical a-C matrix $\left(z_{a m} \simeq 3.2\right)$, and $\mathrm{C}_{168}$ schwarzite is embedded in a low-density matrix $\left(z_{a m} \simeq 2.8\right)$. In the latter cell, the amorphous matrix exhibits a characteristic open nanoporous structure resembling random pore schwarzites $\frac{10.24}{}$ In the polybenzene and PCCM cells, the matrix is more or less homogeneous, in this sense, but characteristic benzenelike and eightfold rings, respectively, can be easily identified. In the diamond cell, it is interesting to note that there is a remarkable tendency of $\mathrm{sp}^{3}$ atoms in the matrix to gather and enrich the interface region around the nanodiamond. A careful inspection of the local structure reveals an increasing degree of crystallinity, indicating that such tetrahedral atoms may act as possible nucleation centers for expanding the size of the diamond nanocrystal under the appropriate conditions. We discuss this further below. 


\section{B. Formation energies}

The central issue of interest in this paper concerns the energetics and stability of the nanostructures. Whether a certain nanocrystal is stable or not can not be told by just comparing the total energy of the composite structure to a reference energy, e.g. diamond. One needs to consider the interaction of the embedded configuration with the host. Here, this is taken care of properly by defining the formation energy of a nanocrystal, given by

$$
E_{\text {form }}=E_{\text {total }}-N_{a} E_{a}-N_{c} E_{c}
$$

where $E_{\text {total }}$ is the total cohesive energy of the composite system (amorphous matrix plus nanocrystal), calculated directly from the simulation, $E_{c}$ is the cohesive energy per atom of the respective crystalline phase, $N_{c}$ is the number of atoms in the nanocrystal, $N_{a}$ is the number of atoms in the amorphous matrix, and $E_{a}$ is the cohesive energy per atom of the pure, undistorted amorphous phase (without the nanocrystal) with coordination $z_{a m}$. A negative value of $E_{\text {form }}$ denotes stability of the nanostructure, a positive value indicates metastability.

To compute $E_{\text {form }}$, we need $E_{a}$ for an arbitrary $z_{a m}$. This is obtained in the following way. We carried out an extensive energetics study of a-C as a function of coordination, or $\mathrm{sp}^{3}$ fraction, over the whole region where a-C is a rigid material. Such a study has not been done before. The results of calculations of the cohesive energy of a number of a-C configurations are plotted in Fig. 3. The minimum of energy is, not surprisingly, at $z_{a m}=3$, where predominance of $\mathrm{sp}^{2}$ bonding is taking place. With increasing coordination, $E_{a}$ becomes higher because the mixed $\left(\mathrm{sp}^{3} / \mathrm{sp}^{2}\right)$ phase is strained ${ }^{13,14}$ This is maximized in the tetrahedral region. On the other side, characterized by configurations with many twofold atoms, the variation is more steep with the maximum energy occuring when the networks become floppy $\left(z_{a m} \simeq 2.4\right) \stackrel{25}{2}$ A cubic polynomial best fits the $z_{a m} \geq 3$ region, while a linear fit applies to the $z_{a m}<3$ region. From these fits one can extract $E_{a}$ for any arbitrary value of $z_{a m}$.

We remind the reader that the absence of $\pi$ repulsion in the potential generates somewhat denser networks in the intermediate region $\left(3.3<z_{a m}<3.6\right)$. This, in turn, overestimates the cohesive energies. Correction of this would slightly shift the energies in this region to higher values, producing a straight line in Fig. 3, but the trends reported here, based on relative energy differences, remain unaltered.

For each one of the six nanostructures considered in this work, we have generated a series of cells with different $z_{a m}$ of the amorphous matrix, but keeping the size of the nanocrystal constant. Then, application of the above methodology yielded $E_{\text {form }}$ as a function of $z_{a m}$. The energy curves resulting from this analysis are shown in Fig. 4. There are a number of important aspects of these results. The first concerns the question of thermodynamic stability. A stable structure has, in principle, the potential to expand against the surrounding matrix, provided that the barriers for this transformation can be overcome. Of the six nanostructures considered, only diamond and the $\mathrm{C}_{168}$ schwarzite are actually stable structures $\left(E_{\text {form }}<0\right)$, the former at high $z_{a m}\left(\rho_{a m} \geq 3 \mathrm{gcm}-3\right)$, the latter at low $z_{a m}\left(\rho_{a m} \leq 1.2 \mathrm{gcm}^{-3}\right)$. With increasing (decreasing) $z_{a m}$ diamonds $\left(\mathrm{C}_{168}\right)$ become more stable, respectively. Yet, diamonds are overall relatively more stable than schwarzites. The enhanced stability of diamonds in highly tetrahedral networks indicates that once nucleation centers are formed, possibly by intense ion irradiation, large diamond regions can be further grown.

The other nanostructures studied in this work are metastable $\left(E_{\text {form }}>0\right)$ through the whole coordination range. However, their curves exhibit a well defined local minimum at which $E_{\text {form }}$ is quite small, suggesting that their synthesis is possible, and that they can be maintained in the amorphous matrix under moderate conditions of temperature and pressure.

Remarkably, there is a reversal of relative stability between these embedded metastable phases, contrasted to their free-standing energetics. Take for example the case of the BC8 structure, which initially is the highest in energy (see Fig. 1) but when embedded in a matrix with $z_{a m} \simeq 3.4$ becomes only slightly metastable. This demonstrates the significance of the interaction between the crystallite and the host. The interaction becomes favorable when the amorphous environment enforces relaxations in the BC8 nanocrystal that shift the bond lengths and angles to more tetrahedral values. Specifically, there are two sets of bond lengths and angles in the BC8 structure: three bonds are $1.62 \AA$ long and one is $1.44 \AA$ long (relaxed with the present potential). The angle subtended by a long and a short bond is $101.5^{\circ}$, and by two long bonds $116.1^{\circ}$. The embedding induces a shift of the short bond to $1.52 \AA$, while the longer bond remains unaltered, and of the small angle to $109^{\circ}$ (the large angle remains the same). A similar reversal in relative stability occurs between polybenzene and PCCM. The latter becomes more stable in the amorphous matrix.

The transition of diamonds from metastability to stability at $z_{a m} \simeq 3.6$ gives us the opportunity to furnish a quantitative definition of tetrahedral a-C (ta-C), vaguely referred to as the form of a-C with a high fraction of $\mathrm{sp}^{3}$ bonding. We define ta-C as the form of a-C with a fraction of $\mathrm{sp}^{3}$ sites above $60 \%$, in which diamond crystallites are stable. In other words, the predominantly tetrahedral amorphous network of ta- $\mathrm{C}$ is able to sustain its crystalline counterpart. Networks with $\mathrm{sp}^{3}$ fractions below $60 \%$ do not belong to the class of ta-C materials, because they can not be transformed into a stable nanocrystalline state. 
One way of checking the stability of nanocrystals, besides referring to $E_{\text {form }}$, is to subject them to thermal annealing. A stable structure should be sustained in the amorphous matrix, while a metastable structure should shrink in favor of the host. We quantitatively demonstrate this by means of the tetrahedral vector $\vec{v}_{t}$, which is the sum of the vectors pointing from an atom to its nearest neighbors, and shows the deviation from ideal tetrahedral geometry. The magnitude of this quantity is plotted in Fig. 5 for diamond crystallites, in both the stable and metastable regions, "as-grown" and after annealing. The nanocrystals have a radius of $\sim 8.5 \AA$. Already before annealing, the metastable nanocrystal is heavily deformed in the outer regions near the interface with the amorphous matrix. The stable one is only slightly deformed. Upon annealing, it is evident that the former structure extensively shrinks, and only a small core remains intact, while the stable structure retains its tetrahedral geometry.

In principle, a stable nanocrystal not only retains its shape and structure upon annealing but, as said earlier, should expand against the amorphous matrix. We have not seen this to happen dynamically in the simulations, which were carried out at relatively high temperatures (1500-2000 K) to accelerate the deterioration of the unstable nanocrystals. Actually, such a thermal annealing experiment, either in the laboratory or on the computer, should take place at temperatures below $1200 \mathrm{~K}$, i.e., in the region where the tetrahedral amorphous network is stable against the transformation of $\mathrm{sp}^{3}$ to $\mathrm{sp}^{2}$ bonding 13 It is doubtful that growth of diamonds will be observed dynamically in simulations at these temperatures because of the enormous computational time needed, but we expect this to happen experimentally.

\section{Phase transformations}

Another interesting aspect of our results is that the formation energies of $6.8^{2} \mathrm{D}$ and PCCM at their minimum (see Fig. 4) are only slightly lower than diamond's at the same $z_{a m}$. This suggests the possibility of ready transformation of $\mathrm{sp}^{2}$-bonded structures to diamond under the appropriate conditions of pressure. To check whether such conditions exist, we calculated the stress fields within the embedded nanocrystals. We found high compressive atomic stresses ${ }^{13}$ in both $6.8^{2} \mathrm{D}$ and PCCM that approach $50 \mathrm{GPa}$ in the center, even at their minimum energy configurations. This is illustrated in Fig. 6 for both cases. The compressive stress reduces as we move radially outwards to the interface. (For $6.8^{2} D$, it remains high up to two thirds of the nanocrystal radius). The origin of the compression is attributed to the lower density and the more open configurations of these nanocrystals compared to the amorphous background. On the other hand, diamond nanocrystals in a ta-C matrix are somewhat denser than the tetrahedral amorphous network around them, and they are found to be under slight tensile stress.

Such huge compressive stresses in the center of the $\mathrm{sp}^{2}$-bonded structures are proper conditions to locally instigate the $\mathrm{sp}^{2} \rightarrow \mathrm{sp}^{3}$ transition and the nucleation of diamond cores, provided that the barriers for the transformation are overcome by means of external pressure. (Compression is the favorable stress state for the formation of $\mathrm{sp}^{3}$ local geometries. ${ }^{13}$ Let us emphasize that the so-produced diamonds would be metastable, because their formation energies in this region are positive. Similar compressive conditions are thought to be responsible for the nucleation of diamond cores in the center of spherical onions under electron irradiation. ${ }^{11}$

\section{Size dependence}

Finally, we checked the stability of the embedded nanocrystals with respect to their size, while keeping the same $z_{a m}$ for the amorphous matrix. An example of this analysis is shown in Fig. 7 for diamonds. The difference between a stable and a metastable nanocrystal is striking. The former has a deep minimum bounded by a steep branch at small radii, indicating a critical size of $\sim 6.5 \AA$, and by a barrier on the right that separates it from other less stable states. Note that, due to the periodic boundary conditions applied to the cells, each crystallite interacts with its neighboring images. This interaction is attractive for certain sizes (distances), e.g. for the minimum and the other low energy states, and repulsive for other (barrier). On the other hand, all states of the second nanocrystal are metastable, and only a shallow local minimum is formed at a larger radius than the stable's one. Its critical size is also larger. These findings suggest that by necessity metastable diamonds are on the average larger, albeit more deformed, than stable diamonds in a ta-C matrix would be. Again, we stress out that metastable diamonds in a low- $z_{a m}$ matrix would be sustained at moderately high temperatures for practical mechanical purposes, but for a better nanostructured material it is preferable to nucleate diamond cores in a ta-C matrix. 


\section{CONCLUSIONS}

In conclusion, we have presented in this paper a method to analyze the stability of nanostructures embedded in an amorphous matrix. This powerful and yet simple approach has yielded detailed information about the energetics and stability of na-C, and gave insight into the interaction of nanocrystals with the amorphous environment. Although the number of representative nanostructures could be extended to include other structures suggested in the literature, we believe that the trends presented here are more general and encompass a wider range of situations relevant to na-C.

The central results of this analysis are: (a) only diamond and schwarzite nanocrystals, among those who have been studied, are stable structures in an amorphous matrix, but not in the whole range of $z_{a m}$. The former are stable for $z_{a m} \geq 3.6$, the latter for $z_{a m} \leq 2.9$; (b) The other structures are metastable through the whole range of $z_{a m}$, but they have low formation energies at well-defined local minima, indicating that can be synthesized and sustained at moderate conditions for practical purposes; (c) There exists a possibility for transformation of the $\mathrm{sp}^{2}$-bonded structures to metastable diamonds.

Future work includes the study of the electronic properties of na-C. It is interesting to investigate whether nanocrystalline inclusions in a-C alter its conductivity through the delocalization of the $\pi$ states ${ }^{2}$ Work toward this goal is in progress.

\section{ACKNOWLEDGMENTS}

We are grateful to Efthimios Kaxiras for granting us access to the hardware resources of his group. This work was partly supported by a IENE $\Delta$ grant No. 99 E $\Delta$ 645, from the Greek General Secretariat for Research and Technology.

${ }^{1}$ For recent reviews of novel carbon nanostructures see S. Subramoney, Adv. Mater. 10, 1157 (1998); F. Banhart, Rep. Prog. Phys. 62, 1182 (1999).

2 J. Robertson, review article in Mat. Sci. Eng. R37, 129 (2002).

3 S.R.P. Silva, review article in Handbook of Thin Film Materials 4 (Academic Press), edited by H.S. Nalwa, p. 403 (2002).

4 D. Vanderbilt and J. Tersoff, Phys. Rev. Lett. 68, 511 (1992).

5 T. Lenosky, X. Gonze, M. Teter, and V. Elser, Nature (London) 355, 333 (1992).

6 M. Keeffe, G.B. Adams, and O.F. Sankey, Phys. Rev. Lett. 68, 2325 (1992).

7 B. Winkler, C.J. Pickard, V. Milman, W.E. Klee, and G. Thimm, Chem. Phys. Lett. 312, 536 (1999).

8 G.A.J. Amaratunga, M. Chhowalla, C.J. Kiely, I. Alexandrou, R. Aharonov, and R.M. Devenish, Nature (London) 383, 321 (1996).

9 P. Milani, M. Ferretti, P. Piseri, C.E. Bottani, A. Ferrari, A. Li Bassi, G. Guizzetti, and M. Patrini, J. Appl. Phys. 82, 5793 (1997).

10 D. Donadio, L. Colombo, P. Milani, and G. Benedek, Phys. Rev. Lett. 83, 776 (1999).

11 F. Banhart and P.M. Ajayan, Nature (London) 382, 433 (1996); M. Zaiser and F. Banhart, Phys. Rev. Lett. 79, 3680 (1997).

12 J. Tersoff, Phys. Rev. Lett. 61, 2879 (1988).

13 P.C. Kelires, Phys. Rev. Lett. 73, 2460 (1994).

14 P.C. Kelires, Phys. Rev. B 62, 15686 (2000).

15 U. Stephan and M. Haase, J. Phys.: Condens. Matter 5, 9157 (1993).

16 H.-P. Kaukonen and R.M. Nieminen, Phys. Rev. Lett. 68, 620 (1992).

17 H.U. Jäger and K. Albe, J. Appl. Phys. 88, 1129 (2000).

18 C. Mathioudakis, P.C. Kelires, Y. Panagiotatos, P. Patsalas, C. Charitidis, and S. Logothetidis, Phys. Rev. B 65, 205203 (2002).

19 G. Kresse and J. Hafner, Phys. Rev. B 47, RC558 (1993); G. Kresse and J. Furthmüller, Comput. Mat. Sci. 6, 15 (1996). We use GGA for the exchange-correlation potential and Vanderbilt-type ultrasoft pseudopotentials. The numerical parameters (cut-off energy, $\vec{k}$-points, etc.) were chosen so that error bars in the calculated lattice constants and energies are of the order of $0.1 \%)$.

${ }^{20}$ I. Alexandrou, H.-J. Scheibe, C.J. Kiely, A.J. Papworth, G.A.J. Amaratunga, and B. Schultrich, Phys. Rev. B 60, 10903 (1999).

21 S. Hirono, S. Umemura, M. Tomita, and R. Kaneko, Appl. Phys. Lett. 80, 425 (2002).

22 M.E. Kozlov, M. Hirabayashi, K. Nozaki, M. Tokumoto, and H. Ihara, Appl. Phys. Lett. 66, 1199 (1995).

23 A.G. Lyapin, V.V. Brazhkin, E.L. Gromnitskaya, S.V. Popova, O.V. Stal'gorova, S.C. Bayliss, and A.V. Sapelkin, Appl. Phys. Lett. 76, 712 (2000).

24 S.J. Townsend, T.J. Lenosky, D.A. Muller, C.S. Nichols, and V. Elser, Phys. Rev. Lett. 69, 921 (1992).

${ }^{25}$ H. He and M.F. Thorpe, Phys. Rev. Lett. 54, 2107 (1985). 


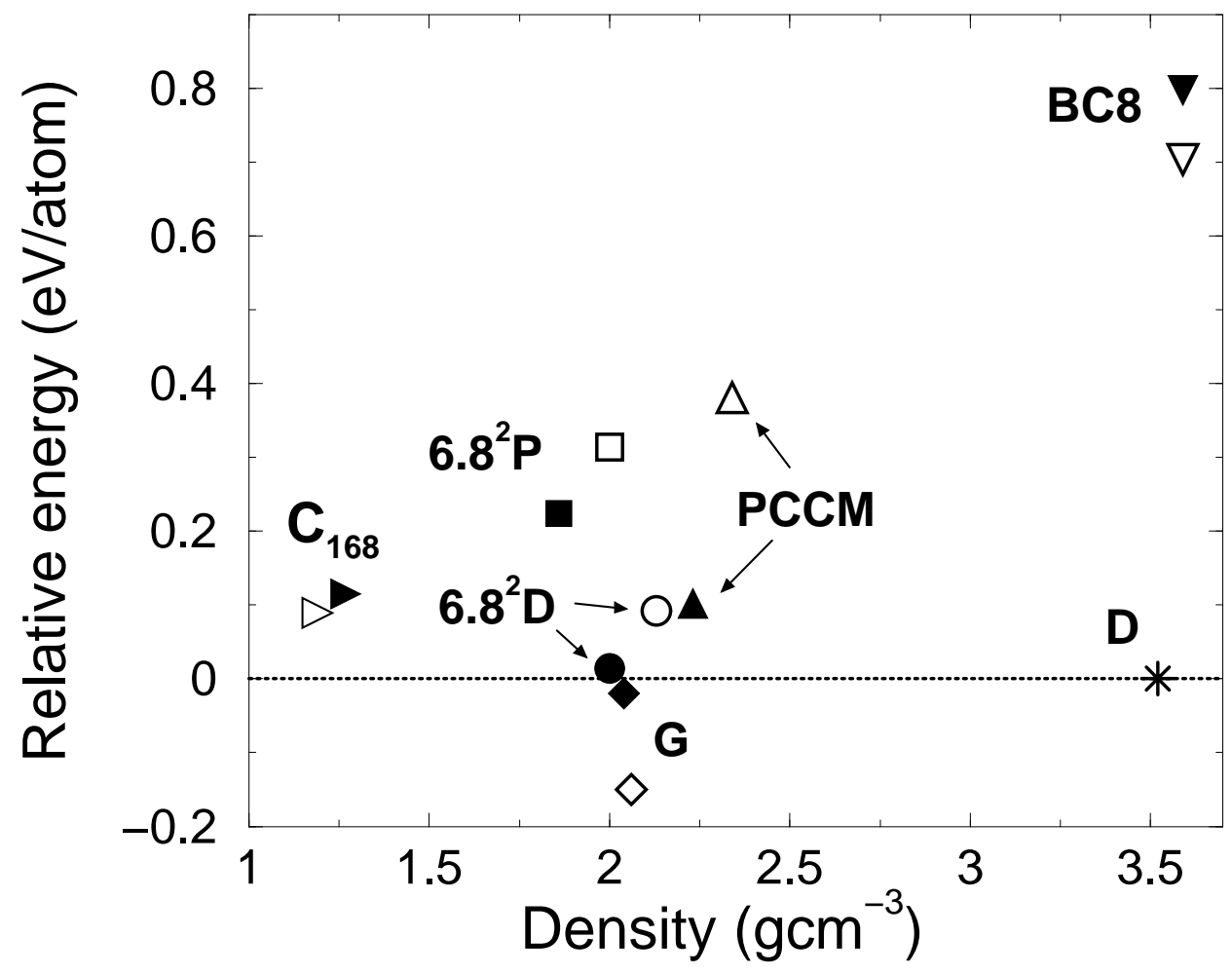

FIG. 1: Energies relative to diamond calculated for various polymorphs of carbon as a function of the density. Filled symbols show results with the Tersoff potential, open symbols denote ab initio results. G stands for graphite. (The empirical result is for a single layer). Other abbreviations are explained in the text. 

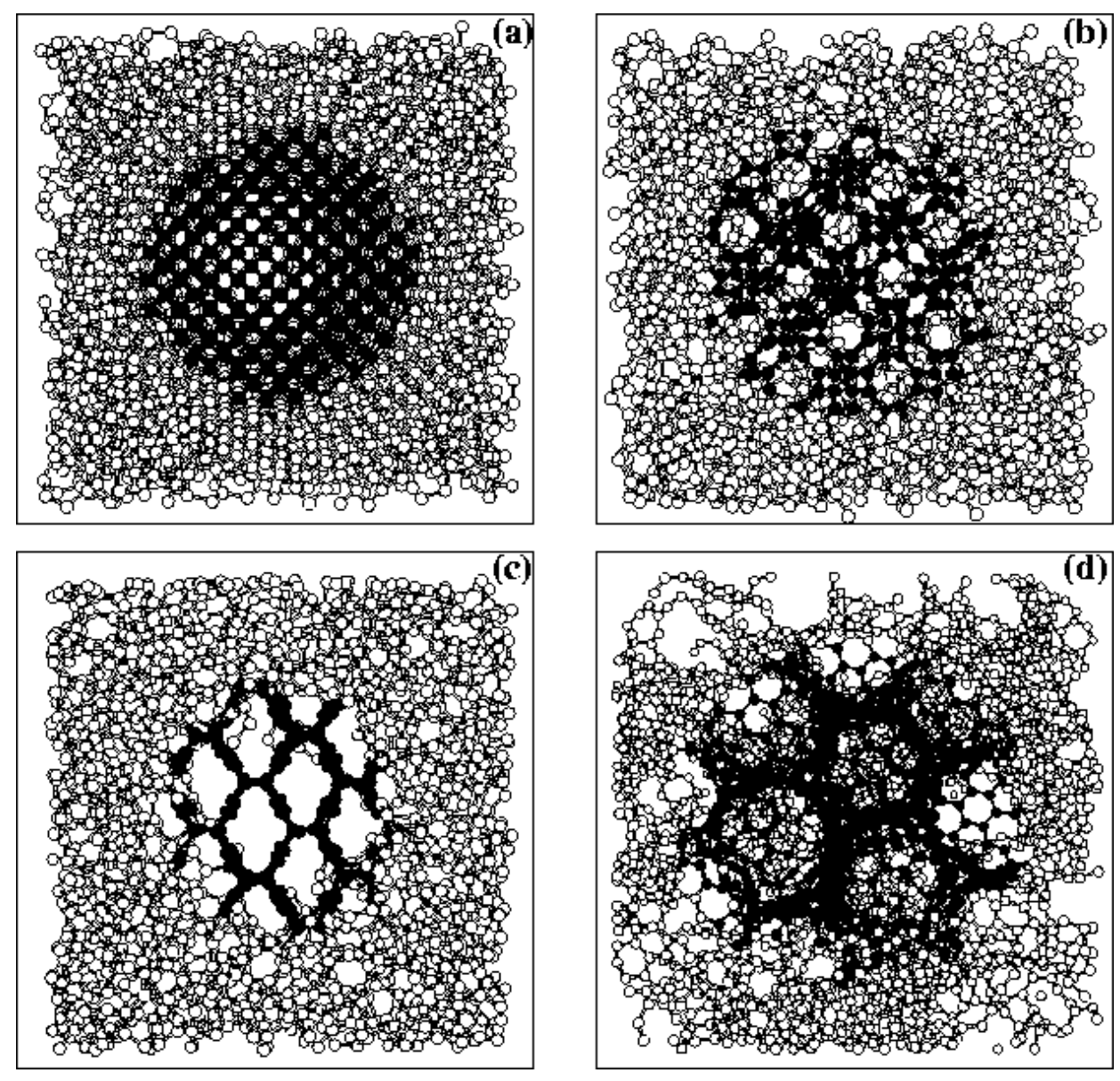

FIG. 2: Cuts from snapshots of four embedded nanostructures: (a) D; (b) $6.8^{2}$ D; (c) PCCM; (d) C168. Open spheres denote atoms in the amorphous matrix. Dark spheres show atoms in the nanocrystals. For clarity, several atoms in the amorphous matrix are not shown to reveal the structures. 


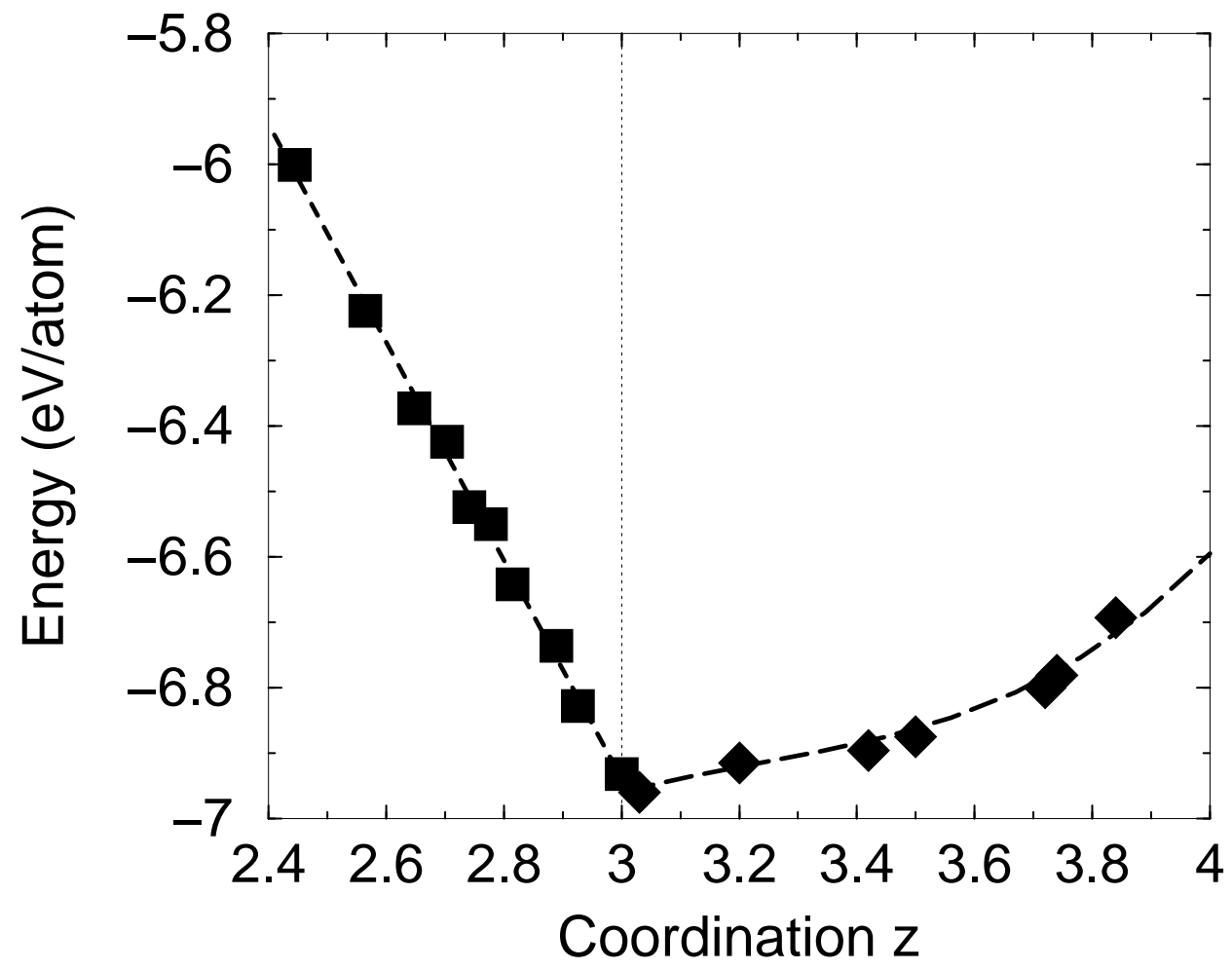

FIG. 3: Cohesive energy versus coordination for a-C configurations. Lines are fits to the points. 

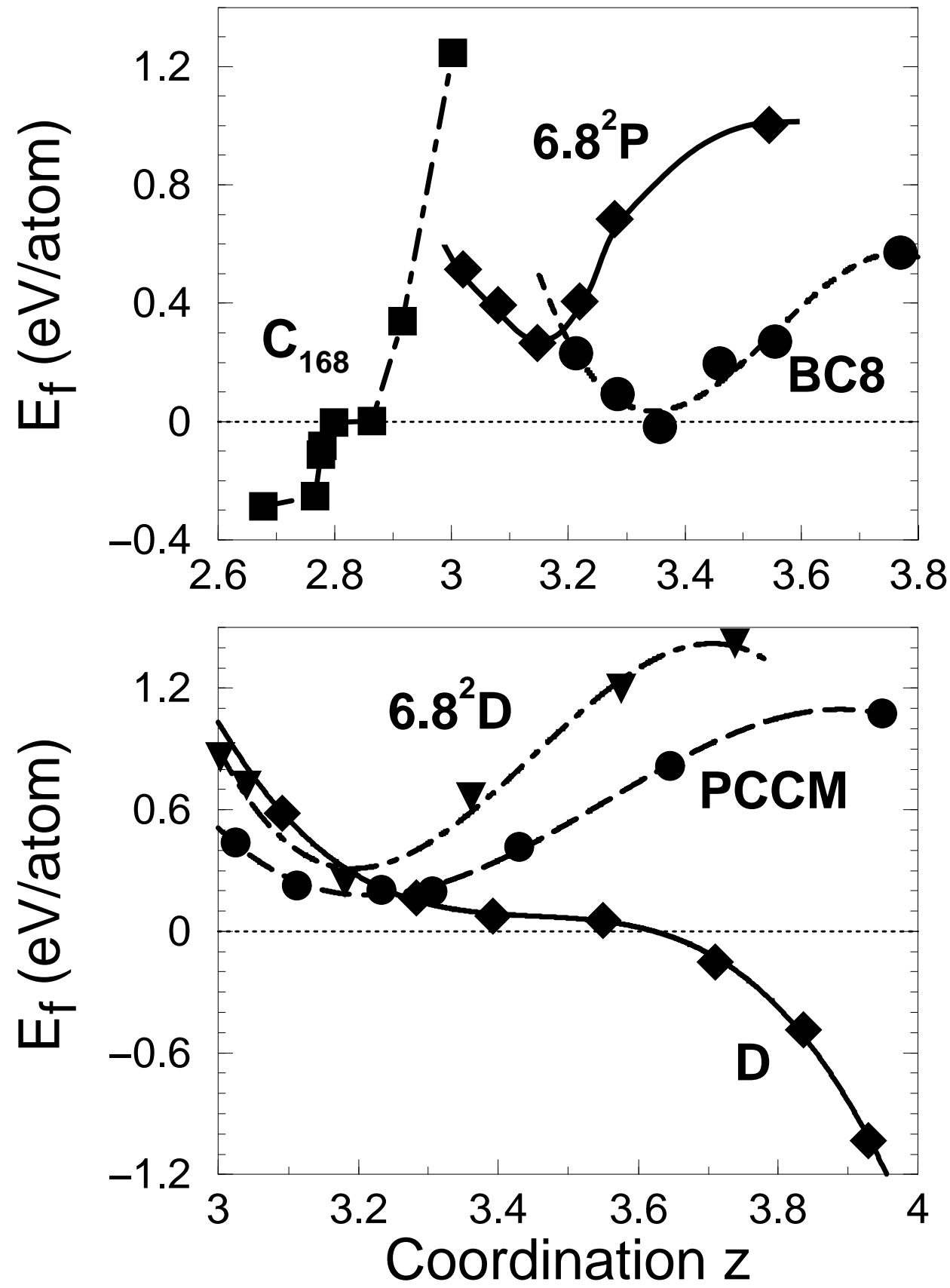

FIG. 4: Formation energies of the six nanostructures considered versus coordination of the amorphous matrix. Lines are fits to the points. 


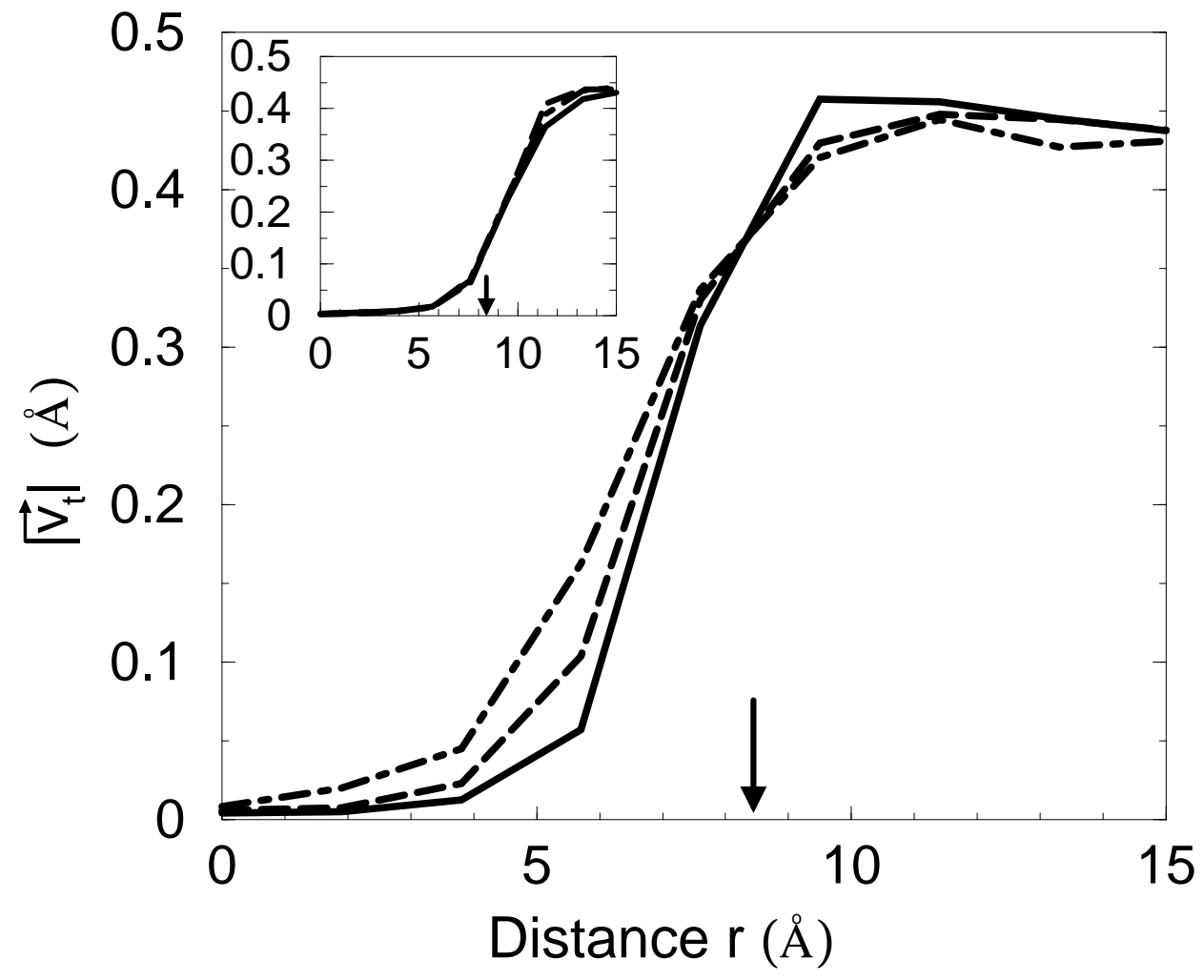

FIG. 5: Variation of the magnitude of the tetrahedral vector as a function of distance from the center of diamond nanocrystals. A zero value indicates ideal tetrahedral geometry. Main figure: metastable case; inset: stable case. Arrows denote position of the interface. Solid line: "as grown"; dashed line: anneal at $1500 \mathrm{~K}$; dash-dotted: anneal at $2000 \mathrm{~K}$. 


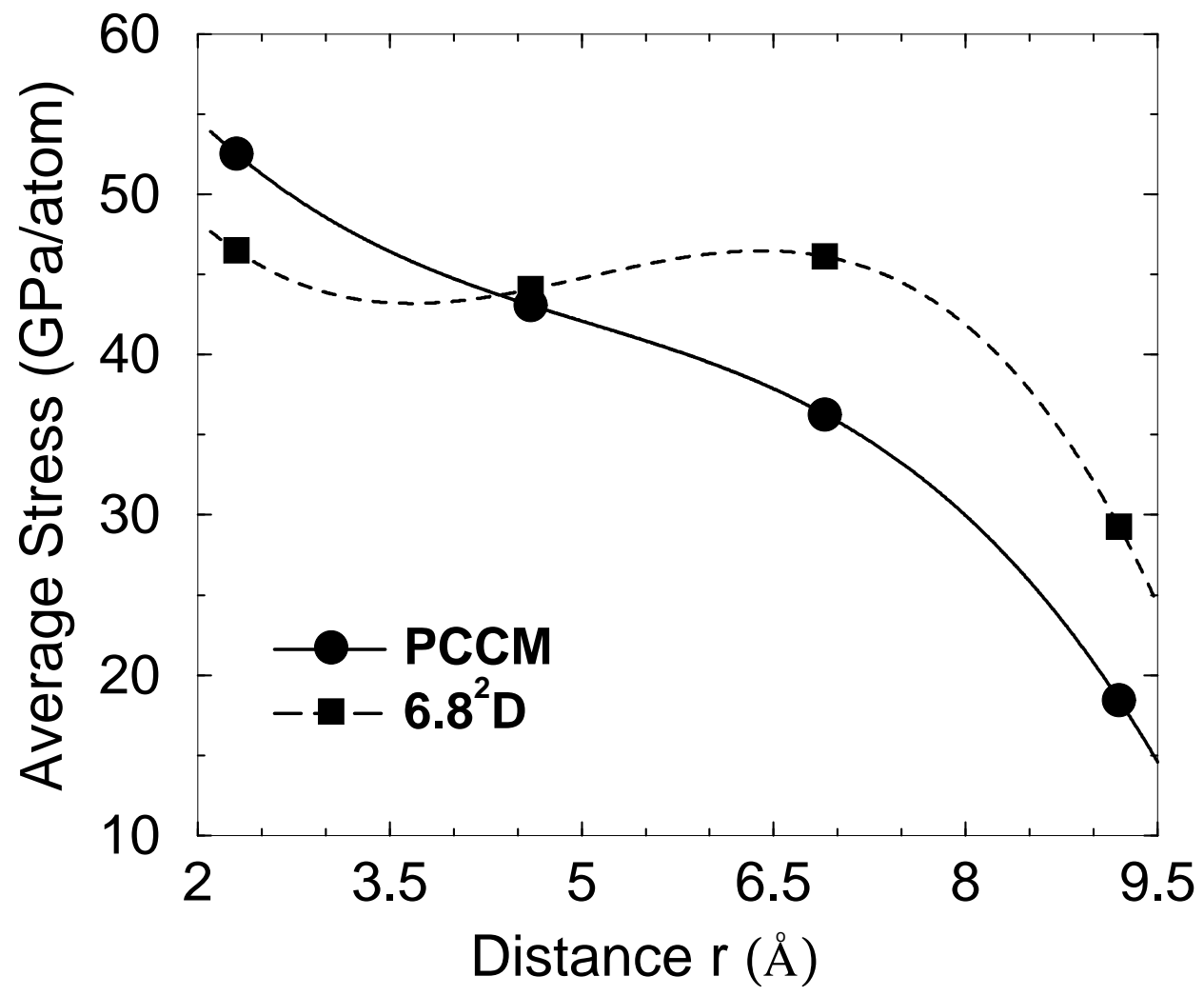

FIG. 6: Atomic stresses in PCCM and $6.8^{2} \mathrm{D}$ nanocrystals, averaged over subsequent spherical shells of width $2.1 \AA$, as a function of thedistance from the center. The stresses are calculated at $300 \mathrm{~K}$. In our formalism [13] positive values denote compressive stresses. Lines are fits to the points.

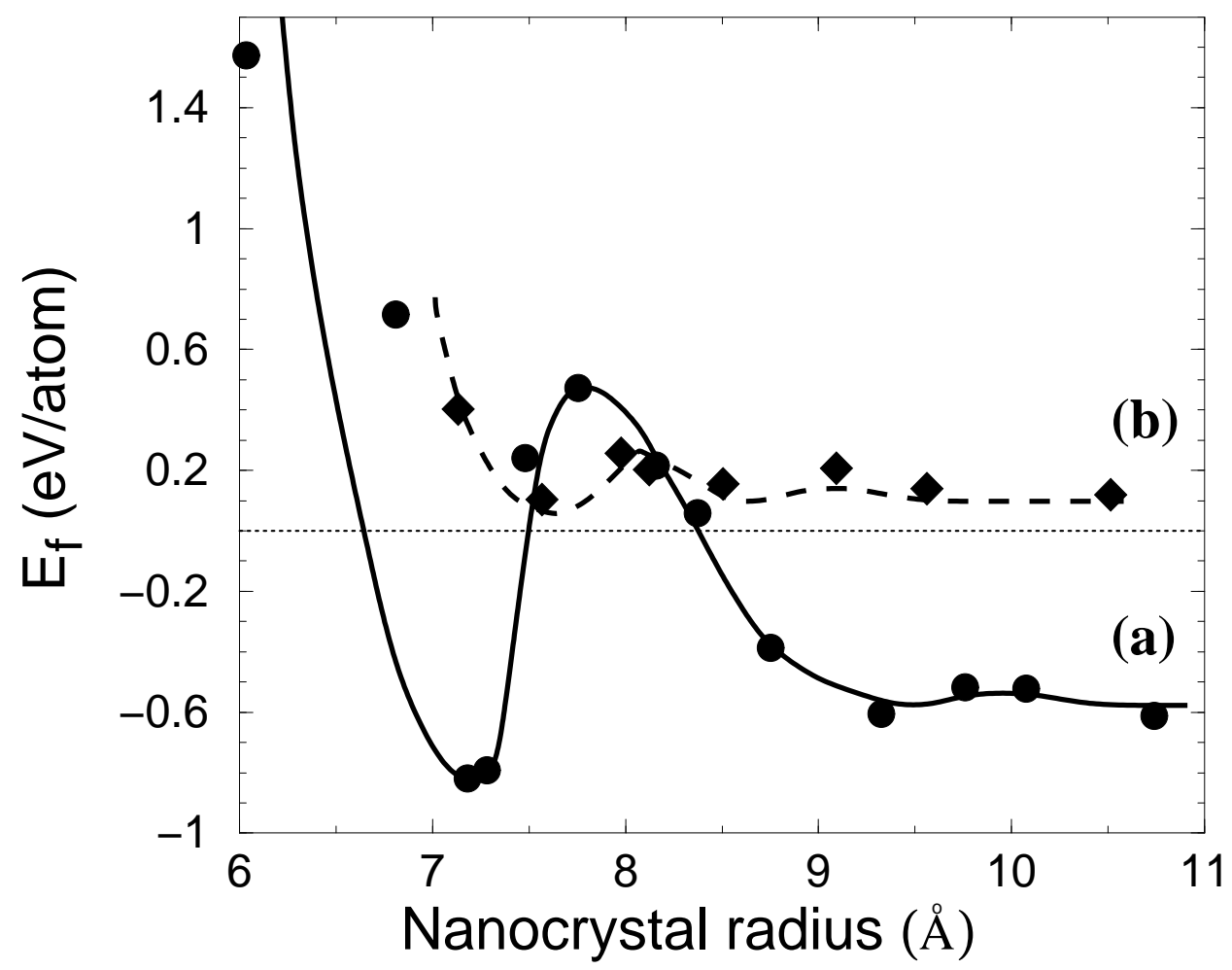

FIG. 7: Formation energy of (a) stable and (b) metastable diamond nanocrystals versus size. 